\title{
Speciation Techniques in Evolved Ensembles with Negative Correlation Learning
}

\author{
Pete Duell, Iris Fermin and Xin Yao
}

\begin{abstract}
The EENCL algorithm [1] has been proposed as a method for designing neural network ensembles for classification tasks, combining global evolution with a local search based on gradient descent. Two mechanisms encourage diversity: Negative Correlation Learning (NCL) and implicit fitness sharing. In order to better understand the success of EENCL, this work replaces speciation by fitness sharing with an island model population structure. We find that providing a population structure that allows for diversity to emerge, rather than enforcing diversity through a similarity penalty in the fitness evaluation, we are able to produce more accurate ensembles, since a more diverse population does not necessarily lead to a more accurate ensemble.
\end{abstract}

\section{INTRODUCTION}

One approach to the design of accurate and diverse ensembles is the Evolutionary Ensembles with Negative Correlation Learning (EENCL) algorithm [1]. Unlike many other ensemble methods, the individual networks are trained in parallel, rather than independently or sequentially. Individual networks learn by Negative Correlation Learning (NCL) [2] and evolutionary learning [3]. Diversity amongst the final population is eforced through the negative correlation of the individual networks' outputs and through speciation by implicit fitness sharing [4][5].

EENCL [1] proved successful on a small number of problems in comparison to other learning algorithms. However, little work has been carried out to analyse why EENCL is effective. Specifically, little analysis has been undertaken to understand the effectiveness of implicit fitness sharing in the algorithm. This paper extends our earlier work [6] on the effectiveness of NCL in the local search of the algorithm and examines the behaviour of the algorithm when implicit fitness sharing is replaced by an island model of subpopulations. Previously we showed that the effectiveness of diversity creation in the local search of the algorithm was negated by the diversity creation of implicit fitness sharing. By replacing implicit fitness sharing we find that we can significantly improve the accuracy of the algorithm on most of the tested datasets. We suggest that this improvement is possible since the new algorithm allows diverse subpopulations to emerge, without enforcing a pre-defined view

Pete Duell is with the School of Computer Science, The University of Birmingham, UK (phone +44 121414 3736; fax +44 121414 4281; email: p.m.duell@cs.bham.ac.uk).

Iris Fermin is with Thales Research \& Technology (UK) Ltd, Reading, UK (phone +44 118986 8601; fax +44 118923 8399; email: iris.fermin@thalesgroup.com).

Xin Yao is with the School of Computer Science, The University of Birmingham, UK (phone +44 121414 3747; fax +44 121414 2799; email: x.yao@cs.bham.ac.uk). on the nature of that diversity. It is not the intention of this work to provide detailed comparisons with other learning algorithms, since this was undertaken with EENCL in Liu and Yao's original work [1]. Here we examine an alternative speciation technique to show that EENCL can be further improved. To our knowledge, this is the first attempt to combine NCL with an island model population structure.

Negative Correlation Learning (NCL) was first proposed by Liu [2] as a means to generate an ensemble of neural networks, whose outputs would be negatively correlated. The networks are trained simultaneously and a penalty term is included during training to encourage the formation of decorrelated networks. Conventional Backpropagation is used to train the network but the error to be minimised is now [7]:

$$
E_{i}=\frac{1}{N} \sum_{n=1}^{N}\left[\frac{1}{2}\left(d(n)-F_{i}(n)\right)^{2}+\lambda p_{i}(n)\right],
$$

where $N$ is number of training patterns, $E_{i}$ is the error of network $i, F_{i}(n)$ is the output of network $i$ on pattern $n, d(n)$ is the target output, $\lambda$ is the strength of penalty parameter and $p_{i}(n)$ is the penalty term, defined for noiseless data as:

$$
p_{i}(n)=\left(F_{i}(n)-d(n)\right) \sum_{j \neq i}\left(F_{j}(n)-d(n)\right) .
$$

NCL seeks to exploit a feature of the bias-variancecovariance decomposition of generalisation error that both bias (squared) and variance are by definition positive, but covariance can be negative:

$$
E=\overline{b i a s}^{2}+\frac{1}{M} v \bar{a} r+\left(1+\frac{1}{M}\right) c o \bar{v} a r,
$$

where bias is the average bias of the networks in the ensemble, $v \bar{a} r$ the average variance, covar the average covariance and $M$ is the number of networks. Reducing the covariance term may however increase the bias term, so NCL seeks to minimise a trade-off between accuracy and negative correlation (diversity).

Liu [7] found that NCL was capable of producing biased individual networks whose errors tend to be negatively correlated on both classification and regression tasks. Brown examined NCL in the wider context of diversity creation techniques, explored bounds on the penalty strength parameter [8][9], and also found that NCL is most effective with less complex networks [10]. Islam used NCL as part 
of a constructive approach to ensemble design, as well as providing a new version of the penalty strength parameter $\gamma$ [11]. The communication costs of NCL have also been reduced through the use of Correlation-Corrected Data [12]. Negative Correlation Learning has also been applied to the creation of fuzzy rules [13].

Evolutionary learning is a population-based approach to solving learning problems. Often the aim is to evolve a single learner, but selecting a single best solution is dependent on the existence of an accurate means of fitness evaluation. In supervised learning tasks the best we can ever obtain is an estimate of how good our learner is based on the performance over the training set (or perhaps a validation set). Since performance over these sets is often a noisy indicator of generalisation performance, especially in the case of overfitting, we are not able to reliably choose the 'best' individual from a population. In fact, Yao showed that a better result can be obtained if we use a number (or even all) of the final population to form an ensemble of learners, as there is more information in the final population than in any single individual [14]. Evolution is also a means to evolve more than the weights of a pre-defined neural architecture: the architecture can itself be evolved [3].

However, the nature of the evolutionary process is such that the population will tend to converge towards similar solutions, as the genetic material of successful individuals is re-used repeatedly to form the new members of the population. In contrast, ensembles are most effective when their members are both accurate and diverse. One mechanism that has been developed to enable evolution to find a diverse set of solutions is speciation through fitness-sharing, originally developed as a means to solve multi-modal optimisation problems through evolution, where a traditional evolutionary approach is liable to concentrate solely on the global maxima [4][5]. Each local maxima is considered to be a niche in the fitness landscape, and fitness sharing encourages different 'species' to evolve, each exploiting a particular niche. Each niche will support a number of individuals proportional to the fitness of that niche. The search for a diverse set of accurate networks can be seen as searching for individual networks that occupy different niches in the fitness space.

The rest of this paper is organised as follows: section II describes the algorithms used in this work; section III describes the experiments conducted; section IV provides our results; section $\mathrm{V}$ conludes the paper and indicates possible future work.

\section{Algorithms}

EENCL uses partial training with the Negative Correlation Learning (NCL) algorithm alongside an evolutionary process to form a population of neural networks suitable for combination into an ensemble [1]. EENCL exploits two mechanisms to ensure that the final networks are both accurate and diverse. Firstly, NCL encourages the negative correlation of the outputs of the networks in the population. Secondly the fitness of individuals in the population is evaluated with implicit fitness sharing [15] based on the coverage of patterns in the training set. Either the entire final population is used to form the ensemble or some subset. In all of the experiments in this paper the entire final population is used. The outputs of the individual networks are combined by either a simple average, majority vote or a winner-takes-all procedure.

The algorithm proceeds according to the following steps [1]:

1) An initial population $(M)$ is trained for a small number of epochs $\left(n_{e}\right)$ according to the NCL algorithm.

2) $n_{b}$ parents are randomly selected from $M$, according to a uniform distribution.

3) $n_{b}$ parents' weights are mutated, by Gaussian mutation $\mathrm{N}(0,1)$ to form $n_{b}$ offspring.

4) $n_{b}$ offspring are added to population and trained for $\mathrm{e}$ epochs, holding the weights of $M$ fixed.

5) Fitness of all $M+n_{b}$ individuals evaluated using a fitness sharing scheme based on the coverage of training patterns.

6) Fittest $M$ individuals from current population $M+n_{b}$ selected for next generation.

7) If the total number of generations reached, go to step 8 , otherwise go to 2 .

8) Combine the population into an ensemble.

Our implementation of the EENCL algorithm differs from Liu's in one respect: we use an amended derivative of the penalty term. Islam et al [11], and later Brown [8], showed that Liu's derivation of the error from equation (1) to give the derivative

$$
\frac{\partial E_{i}(n)}{\partial F_{i}(n)}=(1-\lambda)\left(F_{i}(n)-d(n)\right)+\lambda(F(n)-d(n)),
$$

is flawed and that the correct derivation should be

$$
\begin{aligned}
\frac{\partial E_{i}(n)}{\partial F_{i}(n)} & =\left(F_{i}(n)-d(n)\right) \\
& -2 \gamma\left(1-\frac{1}{M}\right)\left(F_{i}(n)-F(n)\right)
\end{aligned}
$$

where $\gamma$ is now used in place of $\lambda$ as the penalty strength, to distinguish it from Liu's derivation. Conversion from one value to another is possible by means of the equality

$$
\lambda=\gamma\left(2\left(1-\frac{1}{M}\right)\right)=\gamma\left(2 \frac{M-1}{M}\right) .
$$

The learning rate is set to 0.1 and the NCL penalty term $\gamma$ is set at 0.390625 in EENCL and 0.46875 in INCL, in an attempt to provide a comparison to the lambda value of 0.75 in Liu's original experiments [1], according to the equivalence provided in equation 3 .

The raw fitness of an individual is converted to an implicit shared fitness according to:

$$
F_{\text {share }}=\sum_{n} \frac{1}{p_{n}}
$$


where $F_{\text {share }}$ is the shared fitness, $n$ is the training pattern and $p_{n}$ is the number of individuals in the population that correctly classify pattern $n$. This differs from explicit fitness sharing where a sharing radius must be defined to determine if two individuals will share their fitness.

We also introduce a new algorithm, based on EENCL without implicit fitness sharing. Instead the population is divided into sub-populations according to the island model [16]. We term this new algorithm INCL (Island model with Negative Correlation Learning). Each sub-population then evolves in isolation. Migration can occur according to some criteria from one population to another. Such parallel evolutionary algorithms have been used to produce more efficient evolution and also to maintain a diverse set of solutions in numerous studies [17].

INCL differs from EENCL in the following ways. In our implementation, the overall population is divided into equal sized sub-populations. Offspring are trained using NCL to decorrelate their outputs from the other members of the same sub-population. No migration takes place between sub-populations. Fitness is evaluated on raw classification accuracies, with no implicit fitness sharing. At the end of the evolutionary process, the sub-populations are merged into a single ensemble.

\section{EXPERIMENTS WITH BENCHMARK DATASETS}

Liu and Yao [1] showed that the combination of evolution with fitness-sharing and NCL could produce very competitive results in comparison to a number of other classification techniques. Our previous work [6] examined the importance of NCL in the algorithm and found that fitness sharing was the most important factor in determining the diversity and accuracy of the algorithm on the datasets we tested. Here we examine whether it is possible to improve on the performance of EENCL by replacing fitness sharing with a sub-population model (INCL) as an alternative method for encouraging speciation, to test whether fitness sharing is integral to EENCL's performance.

We applied the EENCL and INCL algorithms to four datasets (Australian Credit Card, Pima Indian Diabetes, Heart Disease and Wisconsin Breast Cancer) over 30 independent runs. All of these datasets are available by anonymous ftp from the UCI Machine Learning Repository at ics.uci.edu (128.195.1.1) in directory /pub/machine-learning-databases. Each set was equally divided into a training, validation and testing set. The validation set is not used in these experiments. Each network learns the same training set. The results of all 30 runs on the test set are averaged to approximate the generalisation error of the resulting ensembles. Three different combination schemes are tested: a simple average, majority vote and winner-takes-all. In each case the classification accuracy is shown in table I.

The EENCL algorithm was implemented as described by Liu et al [1]. The initial population is a set of randomly initialised MLP's with full connection and a single hidden layer of 5 nodes. Output nodes are encoded using a 1-of-c scheme and all nodes are sigmoidal logistic of the form

$$
f(x)=\frac{1}{1+\exp (-x)},
$$

where $x$ is the input to the node, and $f(x)$ is the output of the node. The node with the highest output is considered to be the classification of the network. The initial population $M$ is set to 25 , and the number of offspring per generation, $n_{b}$, is 2 . The population is allowed to evolve for 200 generations. Both the initial population and offspring are trained for 5 epochs, $n_{e}$. The learning rate is set to 0.1 and the networks are trained using mean-square-error. The NCL penalty term $\gamma$ is set at 0.390625 , in an attempt to provide a comparison to the $\lambda$ value of 0.75 in Liu's original experiments [1], according to the equivalence provided in equation 3.. This is identical to the experimental setup described by Liu and Yao, arrived at in the original study after limited experimentation [1].

The parameters for INCL are identical to EENCL except that the overall population of 25 is now arbitrarily divided into 5 sub-populations, each with 5 individuals. In this implementation no migration occurs between the subpopulations. It is also worth noting that no attempt has been made to tune the parameters of INCL in order to achieve optimal performance.

\section{RESUlTS}

\section{A. Classification Accuracy}

Classification accuracies for EENCL and INCL can be found in table I. Ensemble outputs are obtained using either simple-average (AVG), majority vote (MAJ) or winner-takesall (WTA) combination scheme. Using a Student t-test with a $1 \%$ confidence interval, INCL has significantly better performance for AVG and MAJ combinations on the Breast Cancer and Heart Disease sets and WTA on the Australian Credit Card datasets (table II). For all other comparisons, no significant difference was found though in each case INCL had a higher accuracy, and in no comparison was EENCL significantly better than INCL.

By replacing fitness sharing in EENCL with an alternative diversity creation technique during global evoultion, classifcation accuracy can be significantly improved for some problems, without significant reduction in others. Our earlier work [6] showed that fitness sharing negates the effects of NCL in EENCL on the problems we tested, and was clearly the most significant aspect of EENCL. This work suggests that the algorithm can be further improved if fitness sharing is replaced by a more suitable speciation technique, such as the island model in INCL.

\section{B. Correlation of Outputs}

Table III gives the average pair-wise correlation between the networks in the ensembles, averaged over the output nodes and 30 runs. The results for the Breast Cancer and Diabetes datasets are significantly different, based on a Student t-test with a $1 \%$ confidence interval (table IV). For the Breast Cancer dataset, a lower correlation was found with the INCL ensemble over the EENCL, but for the Diabetes 
TABLE I

CLASSIFICATION ACCURACIES FOR EENCL AND INCL USING SIMPLE-AVERAGE (AVG), MAJORITY VOTE (MAJ) AND WINNER-TAKES-ALL (WTA) COMBINATION SCHEMES.

\begin{tabular}{|c|c|c|c|c|}
\hline Dataset & Algorithm & AVG & MAJ & WTA \\
\hline Australian Credit & EENCL & 0.876 & 0.873 & 0.867 \\
& INCL & 0.878 & 0.878 & 0.876 \\
\hline Breast Cancer & EENCL & 0.972 & 0.972 & 0.973 \\
& INCL & 0.976 & 0.975 & 0.975 \\
\hline Diabetes & EENCL & 0.766 & 0.764 & 0.762 \\
& INCL & 0.767 & 0.767 & 0.763 \\
\hline Heart Disease & EENCL & 0.789 & 0.785 & 0.774 \\
& INCL & 0.808 & 0.810 & 0.789 \\
\hline
\end{tabular}

TABLE II

PROBABILITY THAT CLASSIFICATION RESULTS ARE FROM THE SAME DISTRIBUTION USING STUDENT T-TEST.

\begin{tabular}{|c|c|c|}
\hline Dataset & Combination & $\mathrm{p}$ \\
\hline Australian Credit & AVG & 0.5155 \\
& MAJ & 0.1007 \\
& WTA & 0.0012 \\
\hline Breast Cancer & AVG & 0.0001 \\
& MAJ & 0.0000 \\
& WTA & 0.0131 \\
\hline Diabetes & AVG & 0.7372 \\
& MAJ & 0.1451 \\
& WTA & 0.7076 \\
\hline Heart Disease & AVG & 0.0032 \\
& MAJ & 0.0002 \\
& WTA & 0.1065 \\
\hline
\end{tabular}

dataset the opposite is true. This is not surprising since both EENCL and INCL attempt to decorrelate the outputs of the networks. However, INCL only decorrelates within a subpopulation, and so there is no guarantee that two networks from different sub-populations will be decorrelated. Further analysis would be interesting to see how correlation differs as the number of sub-populations changes, and also how the correlation in a particular sub-population is affected. It is expected that smaller sub-populations will lead to a lower sub-population correlation, but that this may lead to a higher population correlation, since each network is decorrelated from fewer other networks.

Correlation amongst the final population is significant since the concept that decorrelated (and accurate) networks will reduce generalisation error is the theoretical underpinning for Negative Correlation Learning. NCL attempts to manage the trade off between reducing the bias and covariance terms of the bias-variance-covariance trade-off (equation 2) [18]. Our results show that for different problems EENCL and INCL have differing success in reducing covariance, but that overall, generalisation error is reduced most by INCL. Consequently it appears that INCL is better suited to successfully exploit the trade-off between reducing covariance whilst also maintaining a low bias.
TABLE III

PAIRWISE CORRELATIONS AVERAGED OVER EACH NETWORK AND OUTPUT NODE.

\begin{tabular}{|c|c|c|c|}
\hline Dataset & Algorithm & AVG & STDEV \\
\hline Australian Credit & EENCL & 0.917 & 0.021 \\
& INCL & 0.929 & 0.008 \\
\hline Breast Cancer & EENCL & 0.992 & 0.003 \\
& INCL & 0.984 & 0.003 \\
\hline Diabetes & EENCL & 0.842 & 0.042 \\
& INCL & 0.883 & 0.016 \\
\hline Heart Disease & EENCL & 0.785 & 0.060 \\
& INCL & 0.773 & 0.030 \\
\hline
\end{tabular}

TABLE IV

PROBABILITY THAT CORRELATION RESULTS ARE FROM THE SAME DISTRIBUTION USING STUDENT T-TEST.

\begin{tabular}{|c|c|}
\hline Dataset & $\mathrm{p}$ \\
\hline Australian Credit Card & 0.0001 \\
\hline Breast Cancer & 0.0000 \\
\hline Diabetes & 0.0000 \\
\hline Heart Disease & 0.1828 \\
\hline
\end{tabular}

\section{Joint Correct Sets}

One method to analyse diversity in the final ensemble is to compare their correct response sets [7]. Here we define the correct response set of a network $i, \Omega_{i}$, as the set of examples it correctly classifies. We also define the joint correct response set between networks $i$ and $j, \Omega_{i, j}$, as the set of examples that both networks classify correctly. Table $\mathrm{V}$ shows the mean individual correct response sets, $\Omega_{i}$, and the mean joint correct response sets for all networks in the final population, $\Omega_{\forall i}$, over 30 runs for both EENCL and INCL. Interestingly, whilst our previous work [6] found no significant difference between the size of correct response sets when changing the local search in EENCL, here the results are all found to be significantly different except the joint set for the Breast Cancer dataset, using a Student t-test with a $1 \%$ confidence interval (table VI).

The joint sets for INCL are higher for all the tested datasets, indicating that by this measure EENCL produces more diverse ensembles. This is as expected, since implicit fitness sharing rewards diversity based on the overlap of correct response sets. This does not however necessarily translate into a higher accuracy as seen in table I. EENCL is therefore enforcing a larger degree of diversity than is desirable. INCL on the other hand does not enforce diversity (during evolution) but merely provides a framework where divesity can prosper. These results provide evidence that the latter approach may be preferable, especially when it is unclear how well a particular measure of diversity is suited to a problem.

\section{CONCLUSions}

Our analysis shows that implicit fitness sharing may not be the best way to encourage diverse and accurate evolved 
TABLE V

AVERAGE INDIVIDUAL $\left(\bar{\Omega}_{i}\right)$ AND JOINT $\left(\Omega_{\forall i}^{-}\right)$CORRECT RESPONSE SETS.

\begin{tabular}{|c|c|c|c|}
\hline Dataset & Algorithm & $\bar{\Omega}_{\forall i}$ & $\bar{\Omega}_{i}$ \\
\hline Australian Credit & EENCL & 138.800 & 195.157 \\
& INCL & 160.600 & 197.365 \\
\hline Breast Cancer & EENCL & 219.067 & 225.980 \\
& INCL & 219.733 & 226.548 \\
\hline Diabetes & EENCL & 86.333 & 183.069 \\
& INCL & 144.067 & 192.540 \\
\hline Heart Disease & EENCL & 29.667 & 66.747 \\
& INCL & 37.767 & 68.463 \\
\hline
\end{tabular}

TABLE VI

PROBABILITY THAT CORRECT SET RESULTS ARE FROM THE SAME DisTRIBUTION USING STUDENT T-TEST.

\begin{tabular}{|c|c|c|}
\hline Dataset & Type & $\mathrm{p}$ \\
\hline Australian Credit & $\overline{\Omega_{i}}$ & 0.0000 \\
& $\overline{\Omega_{\forall i}}$ & 0.0000 \\
\hline Breast Cancer & $\overline{\Omega_{i}}$ & 0.0000 \\
& $\Omega_{\forall i}^{-}$ & 0.3827 \\
\hline Diabetes & $\overline{\Omega_{i}}$ & 0.0000 \\
& $\Omega_{\forall i}^{-}$ & 0.0000 \\
\hline Heart Disease & $\overline{\Omega_{i}}$ & 0.0000 \\
& $\overline{\Omega_{\forall i}}$ & 0.0000 \\
\hline
\end{tabular}

ensembles. We find that more accurate ensembles can be produced by providing the conditions for diversity to survive at the global evolutionary level through sub-populations, rather than attempting to enforce a particular definition of diversity within the fitness evaluation. Our results show that in terms of correlation, fitness sharing does not produce more diverse ensembles than the island model in INCL across the datasets we tested. In terms of joint correct sets however, EENCL does produce significantly more diverse ensembles on three out of four datasets as this is the type of diversity that is being encouraged. That this does not translate into improved performance over INCL is evidence that this type of diversity is given too much weight in the EENCL algorithm. We suggest that better results can be obtained by methods that allow diversity to evolve, since they do not attempt to impose a potentially unsuitable predefined concept of 'useful' diversity to an arbitrary problem. It would be helpful to investigate further how successful NCL is in decorrelating the networks in sub-populations of varying sizes. Also it would be interesting to examine how diverse the sub-populations in the algorithm are from one another during evolution. This would be especially important if migration were added to the algorithm.

\section{ACKNOWLEDGMENT}

This work is partially funded by EPSRC, Thales Research \& Technology (UK) Ltd and Advantage West Midlands.

\section{REFERENCES}

[1] Y. Liu, X. Yao, and T. Higuchi, "Evolutionary ensembles with negative correlation learning," IEEE Transactions on Evolutionary Computation, vol. 4, no. 4, pp. 380-387, Nov 2000.

[2] Y. Liu, "Negative correlation learning and evolutionary neural network ensembles," Ph.D. dissertation, University College, The University of New South Wales, Australian Defence Force Academy, Canberra, Australia, 1998.

[3] X. Yao, "Evolving artificial neural networks," in Proceedings of IEEE, vol. 87:9, Sep 1999, pp. 1423-1447.

[4] J. H. Holland, Adaptation in Natural and Artificial Systems. Ann Arbor, MI:University of Michigan Press, 1975.

[5] D. E. Goldberg and J. Richardson, "Genetic algorithms with sharing for multimodal function optimisation," in Proceedings of 2nd International Conference on Genetic Algorithms, J. J. Grefensette, Ed. Hillsdale, NJ: Lawrence Earlbaum, 1987, pp. 41-49.

[6] P. Duell, I. Fermin, and X. Yao, "Diversity creation in local search for the evolution of neural network ensembles," in To appear in Proceedings of 14th European Symposium on Artificial Neural Networks (ESANNO6), 2006.

[7] Y. Liu and X. Yao, "Simultaneous training of negatively correlated neural networks in an ensemble," IEEE Transactions on Systems, Man, and Cybernetics - Part B: Cybernetics, vol. 29, no. 6, pp. 716-725, Dec 1999.

[8] G. Brown, "Diversity in neural network ensembles," Ph.D. dissertation, School of Computer Science, University of Birmingham, 2003. [Online]. Available: citeseer.ist.psu.edu/brown03diversity.html

[9] G. Brown, J. Wyatt, R. Harris, and X. Yao, "Diversity creation methods: A survey and categorisation," Journal of Information Fusion (to appear), 2004.

[10] G. Brown, "On the effectiveness of negative correlation learning," in Proceedings of the First UK Workshop on Computational Intelligence, Q. Shen, Ed., 2001, pp. 57-62.

[11] M. M. Islam, X. Yao, and K. Murase, "A constructive algorithm for training cooperative neural network ensembles," IEEE Transactions on Neural Networks, vol. 14, no. 4, pp. 820-834, Jul 2003.

[12] Z. S. H. Chan and N. Kasabov, "A preliminary study on negative correlation learning via correlation-corrected data (nccd)," Neural Processing Letters, vol. 21, pp. 207-214, 2005.

[13] Y. Cai, X. Sun, and P. Jia, "Negative correlation learning approach for t-s fuzzy models," in Proceedings of 2004 IEEE International Conference on Systems, MAn and Cybernetics, vol. 3, 2004, pp. 22542259.

[14] X. Yao and Y. Liu, "Making use of population information in evolutionary artificial neural networks," IEEE Transactions on Systems, Man and Cybernetics, Part B: Cybernetics, vol. 28, no. 3, pp. 417-425, Jun 1998.

[15] D. E. G. J. Horn and K. Deb, "Implicit niching in a learning classifier system: Nature's way," Evolutionary Computation, vol. 2, no. 1, pp. 37-66, 1994.

[16] T. Back, D. Fogel, and Z. Michalewicz, Handbook on Evolutionary Computation. Oxford University Press, 1997.

[17] E. Cantu-Paz, "A survey of parallel genetic algorithms," Department of Computer Science and Illinois Genetic Algorithms Laboratory, University of Illinois at Urbana-Campaign, Tech. Rep. 91003, 1997.

[18] N. Ueda and R. Nakano, "Generalization error of ensemble estimators," in Proceedings of International Conference on Neural Networks, 1996, pp. 90-95. 